\title{
Comparison of Machine Learning Algorithms for Induction MotorRotor SingleFault Diagnosis using Stator Current Signal
}

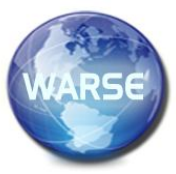

\section{ABSTRACT}

This quantitive researchwas conducted to compare the efficiency between three groups of machine learning' classification techniques for detecting broken rotor bar (BRB)fault in induction motor using stator currents signals with two different signal processing method. Thus, the main purpose of the article is to find out the most suitable method of distributing and extracting data for the fault diagnosis problems. Two of the most common used signal processing method - Discrete Wavelet Transform (DWT) and Fast Fourier Transform (FFT)has been implemented to extract the statiscal features of the faults. Then in the next logical steps, also the most important step, fault diagnosis, three classification algorithms: Support Vector Machines (SVM), K-nearest Neighbors (KNN), and Ensembles are chosen to evaluate the performance and the impact of those different classifiers for induction motor fault diagnosis. Hence, the study found there are five classifiers (Fine Gaussian SVM, Fine KNN, Weighted KNN, Bagged Trees and Subspace $\mathrm{KNN}$ ) are best suited for the proposed problem when providing nearly $100 \%$ classification accuracy for all fault that the other 12 classifiers can not perform well.

Key words: Fault Diagnosis, Induction motor, Stator Current Signal, Discrete Wavelet Transform, Fast Fourier Transform, Machine Learning, Support Vector Machines, K-nearest Neighbors, Ensembles.

\section{INTRODUCTION}

Inductions motors are one of the most commonly used electrical machines in industry because of several technical reasons. They became an industry workhorse and play a pivotal role in industry for conversion of electrical into mechanical energy. Although these electromechanical devices are highly reliable, its susceptible to many types of faults. Such fault can become catastrophic and cause production shutdowns, and even waste of raw materials. Therefore, fault diagnosis of induction motors is very essential in maintaining the continuous operation of industrial processes. Various induction motors fault detection techniques are broadly categorized as 3 main approaches: 1) Signature extraction-based approach; 2) Model-based approach; and 3) knowledge-based approaches [1][2].
The signature-based approaches are achieved by conducting faults signature in time and/or frequency domain. Temperature, vibration, noise, current, voltage, power and even acoustic emission, etc. - all these measurements can be used as monitoring signals. Signatures extracted recorded from those monitoring signal then be used to detect faults. A well-known spectral analysis method of this approaches, Motor Current Signature Analysis (MCSA) has become popular for detection of broken rotor bars or cracked end-rings faults and has attracted concentration of many researchers[2]. However, this method still has some common issues that needs to be improve such as false fault indication. The model-based approaches are based on analytical (i.e. functional) rather than physical redundancy. Thus, the static and/or dynamic relationship i.e. mathematical models to predict behaviors of induction motors under fault conditions[1]. Although model-based approach can estimate the incipient faults by information processing without the need for additional sensors, there is a price for this benefit that results from the need for an accurate through explicit motor models, which may not be always available[2]. Knowledge-based approaches, further more, ultilise deep understanding of the process structure, function and qualitative models under various faulty conditions.Artificial Intelligence techniques have succeeded for both online and offline applications in many electrical systems and devices. Hence, knowledge-based approaches emerged as a promising research direction among them 3 approaches with great potential for industrial implementations with the advanced developments of machine learning algorithms.

Over the two recent decades, the machine learning methods that were most employed for fault diagnosis of induction motors are the artificial neural network (ANN) or hybrid ANN combined with other techniques[3][4][5][6]. Besides, other machine learning algorithms such as the approach associated with Kalman interpolator/ extrapolator[7], the sparse deep learning method which can minimize the risk associated with deep networks[8], ... were also applied.

According to the statistics obtained in several fault diagnosis reports, the most widely used signal is stator current. Some researched on the stator current alone, whereas others reported stator current combined with rotor speed, ... [3][4][5][6][9][10] It could be argued that stator signal is one of the main signals 
used in both knowledge-based approach and signature extraction-based approach.

Although there are various reports on fault diagnosis for induction motors using machine learning based methods, these methods have not been widely applied in practice like other techniques such as MSCA. To utilize the advantages and the intelligent nature of machine learning, practical approaches in industrial applications need to be developed.

To tackle these problems, in this paper, we use the machine learning approach based on calculation signal database which originally recorded by simulation models. Accordingly, the initial signal is stator current, with the Discrete Wavelet Transform (DWT) and Fast Fourier Transform (FFT) being applied in order to construct feature vectors of each class in the database and some classification algorithms such as support vector machine (SVM), K-nearest neighbors $(\mathrm{KNN})$ and Ensemble being chosen as classification algorithms.

The rest of the paper is structured as follow: in part II, we describe the mathematical model and machine learning concept to solve it. In part II, we will provide the machine learning results and compare the efficiency of those classification algorithms. Finally, we conclude the paper in part IV.

\section{REVIEW OF FAULT DIAGNOSIS USING MACHINE LEARNING METHODS}

In this paper, we briefly introduced the concept of machine learning using for fault diagnosis in induction motor, focused on classification tasks, which compares the different accuracies between some popular classification algorithms that are selected. As well as the study of the new deep learning method for this induction motor' faults detection problems. The main idea is illustrated in Fig 1.

Thus, five tasks are needed to implement this method: 1) Build up a simulation model to conduct and record the stator current signals. 2) Choose suitable signal processing method - Discrete Wavelet Transform for features extractions. 3) Extract and calculate features for machine learning. 4) Conduct and compare the classifications" accuracies for electrical faults using chosen classifiers.

\subsection{Simulation Setup}

Each phase of the rotor of induction motor is composed of several bars in parallel. When a certain phase of the rotor bar breaks, it is equivalent to adding a resistance in the fault phase of the rotor. MATLAB Simulink is used to simulate and change the resistance of the fault phase to obtain the original stator current for further analysis as shown in Fig 1.

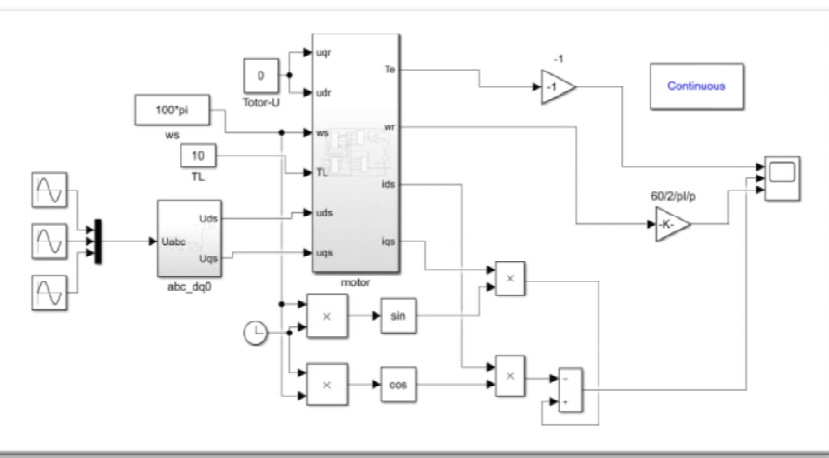

Figure 1:Simulation model of asynchronous motor rotor bar breaking

\subsection{Stator Current}

Recently, signature extraction approach, especially, MCSA and knowledge-based approach using machine learning techniques has received lots of attention and achievements, in particular, motor fault diagnosis. Current - monitoring can be implemented inexpensively on most machines by ultilizing the current transform, which are placed on the motor control centers or switchgear [11]. It's also recognized that the fault pattern in the current signal are unique and cannot be affected by working environments. Many reported have verified the reliability of using current signal for fault diagnosis. Example include air-gap eccentricity[12], stator faults[13], broken rotor bars[14] and motor bearing damage[15]. In this context, we use the stator current signal database to detect and localize only the rotor faults, exactly broken rotor bars (BRB).

\subsection{Discrete Wavelet Transform (DWT)}

Wavelet transform is a powerful tool for multi-scale representation of signals. It can decompose a signal into wavelets confined by both time and frequency. In this study, we use DWT analysis to analyze the initial stator current signal data. The discrete wavelet transforms (DWT) permits a systematic decomposition of a signal into its subband levels as a preprocessing of the system. Thus, the wavelet $\mathrm{db} 4$ is selected as mother wavelets under consideration of $6^{\text {th }}$ level decomposition.

Since different faults have different effects for the stator current, the aiming of DWT processing is to extract statistical of the original signal after signal decomposition. Eight statistical features are determines using DWT as follows: mean, median, standard deviation, median absolute deviation, mean absolute deviation, L1 norm, L2 norm, and maximum norm as tabulated in Table 1.

Table 1: Attributes of Cleveland dataset[16][17]

\begin{tabular}{|c|c|}
\hline Features & Formations \\
\hline Mean & $\begin{array}{l}\mu_{x}=\frac{1}{N} \sum_{i=1}^{N} x_{i}, \text { where } x_{i} \text { is the } \mathrm{i} \text {-th sampled } \\
\text { measurement points, } \mathrm{i}=1,2,3, \ldots, \mathrm{N} \text { for } \mathrm{N} \\
\text { observations. }\end{array}$ \\
\hline Median & $\operatorname{med}=\frac{1}{2}\left(x_{\left(\left[\frac{N+1}{2}\right]\right)}+x_{\left(\left[\frac{N}{2}\right]+1\right)}\right)$ \\
\hline $\begin{array}{l}\text { Standard } \\
\text { Deviation (Std. } \\
\text { Dev.) }\end{array}$ & $\sigma=\sqrt{\frac{1}{N} \sum_{i=1}^{N}\left(x_{i}-\mu_{x}\right)^{2}}$, where $\mu_{x}$ is the mean. \\
\hline $\begin{array}{l}\text { Median } \\
\text { Absolute } \\
\text { Deviation }\end{array}$ & Median_AD $=$ median $\left(\left|x_{i}-\operatorname{median}(X)\right|\right.$ \\
\hline $\begin{array}{l}\text { Mean Absolute } \\
\text { Deviation }\end{array}$ & Mean_AD $=\frac{1}{N} \sum_{i=1}^{N}\left|x_{i}-\mu_{x}\right|$ \\
\hline L1 norm & $\begin{array}{l}\|L\|_{1}=\sum_{i=1}^{N}\left|x_{i}\right| \text {, the sum of absolute values of } \\
\text { its components, also known as one-norm, or mean } \\
\text { norm. }\end{array}$ \\
\hline L2 norm & $\begin{array}{l}\|L\|_{2}=\sqrt{\sum_{i=1}^{N}\left|x_{i}\right|^{2}} \text {, the square root of the sum } \\
\text { of the squares of absolute values of its } \\
\text { components, also known as two-norm, or mean- } \\
\text { square norm. }\end{array}$ \\
\hline $\begin{array}{l}\text { Maximum } \\
\text { norm } \\
\text { (Max norm) }\end{array}$ & $\begin{array}{l}\|L\|_{\infty}=\max \left\{\left|x_{i}\right|: i=1,2,3, \ldots, n\right\}, \\
\text { maximum of absolute values of its components, } \\
\text { also known as infinity norm, or uniform norm. }\end{array}$ \\
\hline
\end{tabular}

Table II above shows an example of that 8 features of stator current $\mathrm{I} 2$ of the second motor with $1 \mathrm{BRB}$ faults load $100 \%$. 


\subsection{Fast Fourier Transform (FFT)}

The Fourier Transform, especially the Fast Fourier Transform, is one of the most popular spectral analysis, and is widely used in several induction motor'sfault detection methods such as MCSA.Some work such as [17][18] have also used this transform to analyze the stator current spectrum for diagnose the broken rotor bars. From these research, internal faults and broken rotor bars may be detected in the stator current spectrum.

In this paper, we use Fast Fourier transform to represent the waveform of the original stator current signal in the frequency domains. Then, the 8 frequency components with maximum amplitude are selected to sample and calculate statistical features. Through FFT-based spectral analysis, eight features defined in Table 1 are also determines.

\section{MACHINE LEARNING RESULTS}

In this paper, 2 machine learning classification algorithms, $\mathrm{KNN}$ and SVM, are selected with 12 different classifiers.

\subsection{The classification algorithms}

SVM is a commonly used machine learning method in data classification and regression based on statistical analyzing and structural risk minimization. This algorithm $\mathrm{h}$ ability to handle the large features spaces as SVM training is performed in such a way that the dimension of the classified vectors does not have any significant effect on the performance of the SVM as well with the performance of other common classifiers[19]. SVM also is generally suitable with separable and non-separable data profile. In such profile, SVM would divide the data into 2 classes: positive and negative then both classes are trained to provide informationabout the classification and builds the hyperplane. Accordingly, the hyperplane maximise the margin of separation between the positive and the negative classes. That is when the soft margine (hyperplane), or the smallest distance between the structure of the separable and nonseparable data set, being used to distinguish the data point. Kernel functions of SVM is selected for non-linear transformation. For example, a kernel function can convert a nonlinearly separable object into linearly separable by mapping them in a higher dimensional feature space. The selection of an appropriate kernel function is critical in the classification process as the kernel defines the feature space in which the training set examples are classified.Linear, polynomial, and radial basis kernels are chosen for this task and its functions are listed in the Table 2 below.

Table 2: Common kernel function

\begin{tabular}{|c|c|c|}
\hline $\begin{array}{c}\text { Kernel } \\
\text { name }\end{array}$ & $\begin{array}{c}\text { Kernel function } \\
\text { formulas }\end{array}$ & \multicolumn{1}{c|}{ Descriptions } \\
\hline $\begin{array}{c}\text { Linear } \\
\text { Kernel }\end{array}$ & $k(x, y)=x^{T} y+C$ & $\begin{array}{l}\text { Linear kernel is the basic } \\
\text { kernel function. It's given by } \\
\text { the inner product }(x, y) \text { plus } \\
\text { an optional constant C. }\end{array}$ \\
\hline $\begin{array}{c}\text { Polynom } \\
\text { ial } \\
\text { Kernel }\end{array}$ & $\begin{array}{l}k(x, y) \\
=\left(\alpha x^{T} y+C\right)^{d} \\
\text { Where, adjustable } \\
\text { parameters are the } \\
\text { slope alpha, the } \\
\text { constant term is C } \\
\text { and the polynomial } \\
\text { degree is d. }\end{array}$ & $\begin{array}{l}\text { Polynomial kernel is a non- } \\
\text { stationary kernel, suited for } \\
\text { problems where all the } \\
\text { training data is normalized. } \\
\text { The most used degree is } \mathrm{d}=2 \\
\text { (quadratic kernel) and d }=3 \\
\text { (cubic kernel) as larger } \\
\text { degree seems overfit for } \\
\text { Machine Learning problems. }\end{array}$ \\
\hline $\begin{array}{c}\text { Gaussian } \\
\text { Kernel }\end{array}$ & $\begin{array}{l}k(x, y) \\
=\exp \left(-\gamma\|x-y\|^{2}\right)\end{array}$ & $\begin{array}{l}\text { In Gaussian kernel, } \gamma \text { plays a } \\
\text { major role in the performance }\end{array}$ \\
\hline
\end{tabular}

( RBF)

Where, $\gamma=1 / 2 \sigma^{2}$ is
an adjustable
parameter and $\| \mathrm{x}$ -
$\mathrm{y} \|$ is denoted as
squared Euclidean
distance between two
features vectors.

of the kernel. If over-estimated,

the exponential will behave almost linearly, and the higherdimensional projection will start to lose its non-linear power.

Beside SVM, KNN is one of the simplest machine learning algorithms that are commonly used in classifying data and learning-based approach. Like SVM algorithms, it can also handle data with various characteristics, including nonlinear, multimodal, and even non-Gaussian data. In that method, the position of the training data is kept fixed (K clusters), then for the new data samples, the distance between the training data and the query data is measured. Then it continued to adjust the $\mathrm{K}$ values until it becomes stable. The optimal $\mathrm{K}$ value finally be used to classify the input data by transforming an anonymous dataset into a known one.

Ensemble is a superior classifier that combines multiple diverse single classifier to boost the prediction accuracy.The main idea of this decision fusion methods is to construct multiple classifiers with different types of features, then ensemble classification results obtained by each classifier based on some predefined rules and achieve final classification result, which is better than the result of a single classifier.

\subsection{Classificaiton Algorithms}

The MATLAB Classification Learner Apps is an application of MATLAB that can trains models to classify data using supervised learning. In this paper two classification algorithms, SVM and KNN are chosen to performed fault diagnosis:

- SVM: linear SVM, quadratic SVM, cubic SVM, fine Gaussian SVM, medium Gaussian SVM, coarse Gaussian SVM.

- $\mathrm{KNN}$ : fine $\mathrm{KNN}$, medium $\mathrm{KNN}$, coarse $\mathrm{KNN}$, cosine $\mathrm{KNN}$, cubic KNN and weighted $\mathrm{KNN}$.

Table 3 below will show description of each classifiers used in paper.

Table 3: Classifiers From Matlab Apps

\begin{tabular}{|c|c|c|}
\hline $\begin{array}{l}\text { Classificatio } \\
\mathrm{n} \text { algorithms }\end{array}$ & $\begin{array}{l}\text { Classifier } \\
\text { types }\end{array}$ & $\begin{array}{lcr}\text { Classifier } & \text { description } & \text { from } \\
\text { MATLAB } & \text { classification } & \text { learner } \\
\text { toolbox } & & \\
\end{array}$ \\
\hline \multirow{6}{*}{$\begin{array}{l}\text { Support } \\
\text { vector } \\
\text { machines } \\
\text { (SVM) }\end{array}$} & $\begin{array}{l}\text { Linear } \\
\text { SVM }\end{array}$ & $\begin{array}{l}\text { Makes a simple linear separation } \\
\text { between classes, using the linear } \\
\text { kernel. The easiest SVM to } \\
\text { interpret. }\end{array}$ \\
\hline & $\begin{array}{l}\text { Quadratic } \\
\text { SVM }\end{array}$ & Use the quadratic kernel. \\
\hline & Cubic SVM & Use the cubic kernel. \\
\hline & $\begin{array}{l}\text { Fine } \\
\text { Gaussian } \\
\text { SVM }\end{array}$ & $\begin{array}{l}\text { Make finely detailed distinctions } \\
\text { between classes, using the } \\
\text { Gaussian kernel with kernel scale } \\
\text { set to sqrt }(\mathrm{P}) / 4 \text { where } \mathrm{P} \text { is the } \\
\text { number of the predictors. }\end{array}$ \\
\hline & $\begin{array}{l}\text { Medium } \\
\text { Gaussian } \\
\text { SVM }\end{array}$ & $\begin{array}{l}\text { Make fewer distinctions than a } \\
\text { Fine Gaussian SVM, using the } \\
\text { Gaussian kernel with kernel scale } \\
\text { set to sqrt }(\mathrm{P}) \text {, where } \mathrm{P} \text { is the } \\
\text { number of the predictors. }\end{array}$ \\
\hline & $\begin{array}{l}\text { Coarse } \\
\text { Gaussian }\end{array}$ & $\begin{array}{l}\text { Make coarse distinctions between } \\
\text { the classes, using the Gaussian }\end{array}$ \\
\hline
\end{tabular}


Thanh-Huong Tran et al., International Journal of Advanced Trends in Computer Science and Engineering, 10(3), May - June 2021, 1509 - 1514

\begin{tabular}{|c|c|c|}
\hline & SVM & $\begin{array}{l}\text { kernel with kernel scale set to } \\
\text { sqrt }(\mathrm{P}) * 4 \text {, where } \mathrm{P} \text { is the number } \\
\text { of predictors. }\end{array}$ \\
\hline \multirow{6}{*}{$\begin{array}{l}\text { Nearest } \\
\text { neighbors' } \\
\text { classifier } \\
(\mathrm{KNN})\end{array}$} & Fine KNN & $\begin{array}{l}\text { Make finely detailed distinctions } \\
\text { between classes, with the number } \\
\text { of neighbors set to } 1 \text {. }\end{array}$ \\
\hline & $\begin{array}{l}\text { Medium } \\
\text { KNN }\end{array}$ & $\begin{array}{l}\text { Make fewer distinctions than a } \\
\text { Fine KNN, with the number of } \\
\text { neighbors set to } 10 \text {. }\end{array}$ \\
\hline & $\begin{array}{l}\text { Coarse } \\
\text { KNN }\end{array}$ & $\begin{array}{l}\text { Make coarse distinctions between } \\
\text { classes, with the number of } \\
\text { neighbors set to } 100 \text {. }\end{array}$ \\
\hline & $\begin{array}{l}\text { Cosine } \\
\text { KNN }\end{array}$ & $\begin{array}{l}\text { Uses a cosine distance metric, with } \\
\text { the number of neighbors set to } 10 \text {. }\end{array}$ \\
\hline & Cubic KNN & $\begin{array}{l}\text { Uses a cubic distance metric, with } \\
\text { the number of neighbors set to } 10 .\end{array}$ \\
\hline & $\begin{array}{l}\text { Weighted } \\
\text { KNN }\end{array}$ & $\begin{array}{l}\text { Uses a distance weighting, with } \\
\text { the number of neighbors set to } 10 \text {. }\end{array}$ \\
\hline \multirow{5}{*}{$\begin{array}{l}\text { Ensemble } \\
\text { classifiers }\end{array}$} & $\begin{array}{l}\text { Boosted } \\
\text { Trees }\end{array}$ & $\begin{array}{l}\text { This model creates an ensemble of } \\
\text { medium decision trees using the } \\
\text { AdaBoost algorithm. Compared to } \\
\text { bagging, boosting algorithms use } \\
\text { relatively little time or memory, } \\
\text { but might need more ensemble } \\
\text { members. }\end{array}$ \\
\hline & $\begin{array}{l}\text { Bagged } \\
\text { Trees }\end{array}$ & $\begin{array}{l}\text { It is a boostrap-agrregated } \\
\text { ensemble of fine decision trees. } \\
\text { Often very accurate, but can be } \\
\text { slow and memory intensive for } \\
\text { large data sets. }\end{array}$ \\
\hline & $\begin{array}{l}\text { Subspace } \\
\text { discriminant }\end{array}$ & $\begin{array}{l}\text { Good for many predictors, } \\
\text { relatively fast for fitting and } \\
\text { prediction, and low on memory } \\
\text { usage, but the accuracy varies } \\
\text { depending on the data. The model } \\
\text { creates an ensemble of } \\
\text { Discriminant classifiers using the } \\
\text { Random Subspace algorithm. }\end{array}$ \\
\hline & $\begin{array}{l}\text { Subspace } \\
\text { KNN }\end{array}$ & $\begin{array}{l}\text { Good for many predictors. The } \\
\text { model creates an ensemble of } \\
\text { nearest-neighbor classifiers using } \\
\text { the Random Subspace algorithm. }\end{array}$ \\
\hline & $\begin{array}{l}\text { RUSBooste } \\
\text { d Trees }\end{array}$ & $\begin{array}{l}\text { Used for skewed data with many } \\
\text { more observations of one class. }\end{array}$ \\
\hline
\end{tabular}

\subsection{Faults Diagnosis Results}

In this part, we use the classification algorithm SVM, KNN and Ensembles to do fault diagnosis and compare the accuracy of algorithms. Two table 5 and 6 below show the average accuracy of all algorithms with different load.
Table 5: Accuracy For Classification For Brb Fault At $10 \%$ Loading Using Various Classifiers

\begin{tabular}{|c|c|c|c|}
\hline \multirow{2}{*}{$\begin{array}{l}\text { Classification } \\
\text { Algorithms }\end{array}$} & \multirow{2}{*}{ Sub-groups } & \multicolumn{2}{|c|}{ Accuracy } \\
\hline & & DWT & FFT \\
\hline \multirow{6}{*}{ SVM } & Linear SVM & 73.8 & 37.5 \\
\hline & Quadratic SVM & 85.7 & 97.6 \\
\hline & Cubic SVM & 97.6 & 100 \\
\hline & Fine Gaussian SVM & 100 & 100 \\
\hline & $\begin{array}{l}\text { Medium Gaussian } \\
\text { SVM }\end{array}$ & 92.5 & 94.6 \\
\hline & Coarse Gaussian SVM & 87.5 & 75 \\
\hline \multirow{6}{*}{$\mathrm{KNN}$} & Fine KNN & 100 & 100 \\
\hline & Medium KNN & 50 & 51.3 \\
\hline & Coarse KNN & 12.5 & 16.7 \\
\hline & Cosine KNN & 45.7 & 52.6 \\
\hline & Cubic KNN & 52.4 & 50 \\
\hline & Weighted KNN & 100 & 100 \\
\hline \multirow{5}{*}{ Ensemble } & Boosted Trees & 12.5 & 12.5 \\
\hline & Bagged Trees & 100 & 100 \\
\hline & Subspace Discriminant & 75 & 16.7 \\
\hline & Subspace KNN & 100 & 100 \\
\hline & RUSBoosted Trees & 12.5 & 12.5 \\
\hline
\end{tabular}

Table 6: Accuracy For Classification For Brb Fault At $30 \%$ Loading Using Various Classifiers

\begin{tabular}{|l|l|c|c|}
\hline \multirow{4}{*}{$\begin{array}{l}\text { Classification } \\
\text { Algorithms }\end{array}$} & \multirow{2}{*}{ Sub-groups } & \multicolumn{2}{|c|}{ Accuracy } \\
\cline { 2 - 4 } & & DWT & FFT \\
\hline \multirow{5}{*}{ SVM } & Linear SVM & 63.7 & 50 \\
\cline { 2 - 4 } & Quadratic SVM & 73.96 & 92.5 \\
\cline { 2 - 4 } & Cubic SVM & 99.8 & 9.6 \\
\cline { 2 - 4 } & Fine Gaussian SVM & $\mathbf{1 0 0}$ & $\mathbf{1 0 0}$ \\
\cline { 2 - 4 } & $\begin{array}{l}\text { Medium Gaussian } \\
\text { SVM }\end{array}$ & 87.5 & 98.2 \\
\cline { 2 - 4 } & Coarse Gaussian SVM & 77.78 & 62.5 \\
\hline \multirow{5}{*}{ KNN } & Fine KNN & $\mathbf{1 0 0}$ & $\mathbf{1 0 0}$ \\
\cline { 2 - 4 } & Medium KNN & 52.26 & 52.4 \\
\cline { 2 - 4 } & Coarse KNN & 17.6 & 12.5 \\
\cline { 2 - 4 } & Cosine KNN & 48.61 & 47.3 \\
\cline { 2 - 4 } & Cubic KNN & 51.39 & 42.5 \\
\cline { 2 - 4 } & Weighted KNN & $\mathbf{1 0 0}$ & $\mathbf{1 0 0}$ \\
\hline \multirow{5}{*}{ Ensemble } & Boosted Trees & 16.7 & 12.5 \\
\cline { 2 - 4 } & Bagged Trees & $\mathbf{1 0 0}$ & $\mathbf{1 0 0}$ \\
\cline { 2 - 4 } & Subspace Discriminant & 87.5 & 16.7 \\
\cline { 2 - 4 } & Subspace KNN & $\mathbf{1 0 0}$ & $\mathbf{1 0 0}$ \\
\cline { 2 - 4 } & RUSBoosted Trees & & 12.5 \\
\hline
\end{tabular}

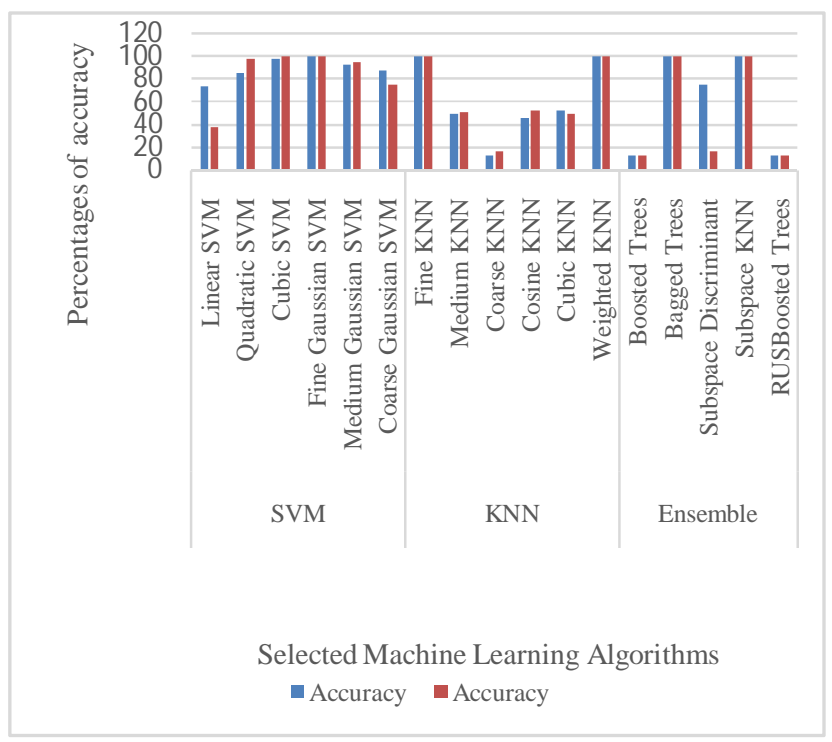

Figure 2: Classification accuracy for BRB fault at load 10\% for all 1512 chosen classifiers 
The results shown at those two figure 2 and 3 that there are 5 best classification functionsfor both data using DWT method and FFT method: Fine Gaussian SVM, Fine KNN, and Weighted KNN, Bagged Trees and Subspace KNN which give the classification accuracy of almost $100 \%$ for all faults for induction motors. However, not every algorithm chosen to be applied in fault diagnosis is suitable. In the worst case, the classification accuracy of Coarse KNN, Boosted Trees and RUSBoosted Trees is only $12.5 \%$. Further, as we just only focus on one type of single faults, so the classification accuracy still not very high, most of them only about 50 $80 \%$.

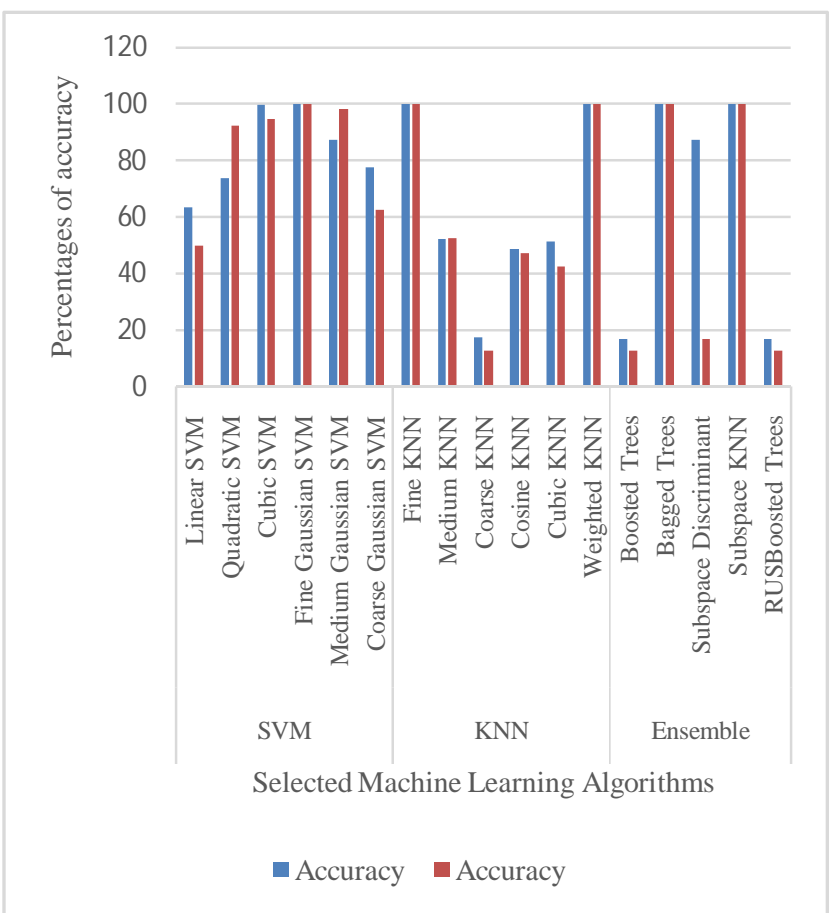

Figure 3: Classification accuracy for BRB fault at load 30\% for all chosen classifiers

The results also show that signals trained using DWT and FFT have higher accuracy when applying the SVM functions rather than the KNN functions, and FFT has better accuracy than DWT for the most SVM classifiers.

\section{CONCLUSION}

In this paper, we suggest and analyse the efficiency of the machine learning algorithm in classification when applying in fault diagnosis with the stator current as the original signal,the Discrete Wavelet Transform (DWT) and Fast Fourier Transform (FFT) are being chosen for feature extraction. However, this is only the initial result of the model when using 3 machine learning algorithms SVM, KNN and Ensembles. According to the obtained results, we can see that: stator current can be used to detect the similar types of faults in different levels of load with almost similar result. Besides, the data set that have been extracted using DWT method is suitable for SVMs than the ones using FFT method. Among the classification functions, the functions give the best efficiency are Fine Gaussian SVM, Fine KNN and Weighted KNN with $100 \%$ efficient.In the future, multiple-faults diagnogis problem will be researched in order to minimize the posibility of failure for induction motor application, especially, electromechanical systems.

\section{ACKNOWLEDGEMENTS}

This work was supported in part by ShanghaiPujiang Program, and in part by Belt andRoad InternationalCooperation Project.

\section{REFERENCES}

[1] K. Kim and A. G. Parlos, "Model-based fault diagnosis of induction motors using non-stationary signal segmentation," Mech. Syst. Signal Process., 2002, doi: 10.1006/mssp.2002.1481.

[2] M. Z. Ali, M. N. S. K. Shabbir, X. Liang, Y. Zhang, and T. Hu, "Machine learning-based fault diagnosis for singleand multi-faults in induction motors using measured stator currents and vibration signals," IEEE Trans. Ind. Appl., vol. 55, no. 3, pp. 2378-2391, 2019, doi: 10.1109/TIA.2019.2895797.

[3] F. Filippetti, G. Franceschini, and C. Tassoni, "Neural networks aided on-line diagnostics of induction motor rotor faults," IEEE Trans. Ind. Appl., 1995, doi: 10.1109/28.395301.

[4] S. Farag, B. K. Lin, T. G. Habetler, and J. H. Schlag, "An Unsupervised, On-Line System for Induction Motor Fault Detection Using Stator Current Monitoring," IEEE Trans. Ind. Appl., 1995, doi: 10.1109/28.475698.

[5] H. Nejjari and M. E. H. Benbouzid, "Monitoring and diagnosis of induction motors electrical faults using a current Park's vector pattern learning approach," IEEE Trans. Ind. Appl., 2000, doi: 10.1109/28.845047.

[6] T. Boukra, A. Lebaroud, and G. Clerc, "Statistical and neural-network approaches for the classification of induction machine faults using the ambiguity plane representation," IEEE Trans. Ind. Electron., 2013, doi: 10.1109/TIE.2012.2216242.

[7] O. Ondel, E. Boutleux, E. Blanco, and G. Clerc, "Coupling pattern recognition with state estimation using Kalman filter for fault diagnosis," IEEE Trans. Ind. Electron., 2012, doi: 10.1109/TIE.2011.2181133.

[8] C. Sun, M. Ma, Z. Zhao, and X. Chen, "Sparse Deep Stacking Network for Fault Diagnosis of Motor," IEEE Trans. Ind. Informatics, 2018, doi: 10.1109/TII.2018.2819674.

[9] F. Ben Abid, S. Zgarni, and A. Braham, "Distinct bearing faults detection in induction motor by a hybrid optimized SWPT and aiNet-DAG SVM," IEEE Trans. Energy Convers., 2018, doi: 10.1109/TEC.2018.2839083.

[10] W. F. Godoy, I. N. da Silva, A. Goedtel, R. H. C. Palácios, and T. D. Lopes, "Application of intelligent tools to detect and classify broken rotor bars in threephase induction motors fed by an inverter," IET Electr. Power Appl., 2016, doi: 10.1049/iet-epa.2015.0469.

[11] T. Han, B. S. Yang, W. H. Choi, and J. S. Kim, "Fault diagnosis system of induction motors based on neural network and genetic algorithm using stator current signals," Int. J. Rotating Mach., 2006, doi: 10.1155/IJRM/2006/61690.

[12] W. T. Thomson, D. Rankin, and D. G. Dorrell, "On-line current monitoring to diagnose airgap eccentricity in large three-phase induction motors - industrial case histories verify the predictions," IEEE Trans. Energy Convers., 1999, doi: 10.1109/60.815075.

[13] S. Williamson and K. Mirzoian, "ANALYSIS OF CAGE INDUCTION MOTORS WITH STATOR WINDING FAULTS.," IEEE Trans. power Appar. Syst., 1985, doi: 10.1109/TPAS.1985.319221. 
[14]W. T. Thomson and M. Fenger, "Current signature analysis to detect induction motor faults," IEEE Ind. Appl. Mag., 2001, doi: 10.1109/2943.930988.

[15] R. G. Bartheld, T. G. Habetler, and F. Kamran, "Motor Bearing Damage Detection Using Stator Current Monitoring," IEEE Trans. Ind. Appl., 1995, doi: $10.1109 / 28.475697$.

[16] J. J. Saucedo-Dorantes, M. Delgado-Prieto, R. A. Osornio-Rios, and R. De Jesus Romero-Troncoso, "Multifault Diagnosis Method Applied to an Electric Machine Based on High-Dimensional Feature Reduction," IEEE Trans. Ind. Appl., 2017, doi: 10.1109/TIA.2016.2637307.

[17] P. Shi, Z. Chen, Y. Vagapov, and Z. Zouaoui, "A new diagnosis of broken rotor bar fault extent in three phase squirrel cage induction motor," Mech. Syst. Signal Process., 2014, doi: 10.1016/j.ymssp.2013.09.002.
[18]M. Y. Kaikaa and M. Hadjami, "Effects of the simultaneous presence of static eccentricity and broken rotor bars on the stator current of induction machine," IEEE Trans. Ind. Electron., 2014, doi: 10.1109/TIE.2013.2270216.

[19] S. Pan, T. Han, A. C. C. Tan, and T. R. Lin, "Fault Diagnosis System of Induction Motors Based on Multiscale Entropy and Support Vector Machine with Mutual Information Algorithm," Shock Vib., 2016, doi: 10.1155/2016/5836717.

[20] K. R. Müller, S. Mika, G. Rätsch, K. Tsuda, and B. Schölkopf, "An introduction to kernel-based learning algorithms," IEEE Transactions on Neural Networks. 2001, doi: 10.1109/72.914517. 